\title{
HACIA UNA VISIÓN INTEGRADORA DE LA PRAGMÁTICA INTERCULTURAL EN EL AULA DE ELE
}

\author{
NOEMÍ DOMÍNGUEZ GARCÍA \\ Universidad de Salamanca \\ YERAY GONZÁLEZ PLASENCIA ${ }^{1}$
}

Universidad de Salamanca

\section{Resumen}

Este trabajo tiene por objetivo ofrecer una concepción de la cultura que favorezca su integración en el currículo de ELE. Para ello, partimos de la antropología simbólica y seleccionamos las unidades que pertenecen a la cultura comunicativa, es decir, aquellos hechos propios de una comunidad cultural que determinan la eficacia comunicativa en sus interacciones. En base a esta premisa, se ofrece una estructura a través de las funciones comunicativas que permita la nivelación de estos contenidos y su aplicación a la práctica docente. Palabras clave: pragmática intercultural, funciones comunicativas, currículo, cultura comunicativa, patrones comunicativos.

\begin{abstract}
This work aims to show a vision of the culture which helps it to integrate SFL curriculum. For this purpose, we begin with the Symbolic Anthropology ad later on we will choose the units which belong to the communicative culture, meaning those own habits from a cultural community that determine the communicative efficiency in their interactions. Based on this, we will offer a structure based on the communicative functions as a tool for leveling these contents in the teaching procedure.
\end{abstract}

Keywords: intercultural Pragmatics, communicative functions, curriculum, communicative culture, patterns of communication.

\section{INTRODUCCIÓN}

Lengua y cultura se relacionan de una manera indisociable. Mucho antes de la aparición de los dos documentos principales que rigen la enseñanza de español

\footnotetext{
${ }^{1}$ Universidad de Salamanca. Correo: noedom@usal.es; yeraigoncia@usal.es. Recibido: 04-06-2015.Aceptado: 15-07-2015. Trabajo realizado en el marco de los proyectos de investigación "Repertorio de eventos comunicativos del habla castellanoleonesa. Aplicación a la enseñanza del español como lengua extranjera para hablantes con árabe e inglés como L1" (Q3718001E), financiado por el Fondo Social Europeo, el Programa Operativo de Castilla y León y la Junta de Castilla y León a través de la Consejería de Educación e "IDELE: Innovation and Development in Spanish as a Second Language" (ref. 530459-TEMPUSI-2012-1-ES-TEMPUS-JPCR), subvencionado por la EACEA en la convocatoria Tempus IV.
} 
como lengua extranjera, el Marco Común Europeo de Referencia para las Lenguas (2002, en adelante, MCERL) y el Plan Curricular del Instituto Cervantes (2006, en adelante PCIC), investigadores de muy variadas disciplinas trataron de esclarecer los fundamentos de esta interdependencia. De este modo, desde la Filosofía del Lenguaje ya se establecieron puentes en siglo XVIII entre el lenguaje y la cultura al considerar el primero como el "órgano que forma el pensamiento" (Humboldt, 1990: 74), es decir, el elemento que permite la conceptualización de lo pensado.

Desde Franz Boas, la Antropología Lingüística también tiene como objeto de estudio la relación lengua-cultura, y si bien en la actualidad ha virado hacia un enfoque más antropológico - puesto que concibe los hablantes como actores sociales y el lenguaje como una herramienta para describir la cultura de ese grupo (Duranti, 2000)-, en el siglo XX, Gumperz y Bennett habían retomado la idea del relativismo lingüístico (Sapir, 1949; Whorf, 1956) afirmando, pese a que consideran que la mayoría de las premisas están desfasadas, que

muchas ideas que consideramos naturales y que aceptamos sin poner jamás en cuestión pueden estar directamente determinadas por nuestros presupuestos lingüísticos (Gumperz y Bennett, 1981: 100).

La Antropología Cultural, como no podía ser de otra manera, ha dado lugar a profundas teorías sobre qué es cultura y qué no². Harris (1968) entiende que en cualquier cultura prima la parte material, como los mecanismos de producción y reproducción, sobre la parte intelectual o conductual, donde se sitúa el lenguaje. Geertz (1973 [1992]), sin embargo, entiende la cultura como un conjunto de símbolos que operan en todas las caras de la sociedad y cuyo significado es accesible únicamente a los miembros de ese grupo. Desde la óptica de la comunicación intercultural, Hofstede $(1980,1999)$ considera la cultura como una cebolla: las capas más externas son las más permeables y susceptibles al cambio mientras que las interiores se mantienen invariables durante el tiempo. De esta manera, en el núcleo se sitúan los valores de las comunidades, los cuales influyen en los modelos interactivos de los hablantes.

Ante este panorama, en nuestra disciplina asumimos que no se puede aprender una lengua sin aprender también su cultura, puesto que el aprendizaje de un idioma no consiste únicamente en la apropiación de un código, sino también de unos conocimientos respecto a los modos de ser, de actuar y de pensar de la población que utiliza ese sistema. Guillén Díaz habla de una 'gramática cultural oculta', la cual

si no se domina, da lugar en la comunicación a casos de incomprensión, conflicto, choques culturales y malentendidos. Se pone en evidencia que la simple adquisición del sistema lingüístico no asegura la comprensión, por lo que no es posible disociar en el plano de la comunicación la lengua de la realidad que subyace, es decir del bagaje cultural (Guillén Díaz, 2004: 838).

Es decir, el plano lingüístico acarrea, inexorablemente, el conocimiento del entorno en el que se pone en práctica el lenguaje, entre otras cosas debido a que el idioma actúa como vehículo de transmisión de una cultura. Sin embargo, a menudo se

${ }^{2}$ Una buena introducción al tema se puede encontrar en Kahn (1975). 
obvia la relación que se establece en dirección opuesta: la manera en que una comunidad categoriza, interpreta y comprende la realidad se ve influida por su cultura de origen y, dentro de esta realidad se encuentra, indiscutiblemente, la comunicación.

Esta imbricación entre lengua y cultura afecta a todos los planos de estudio del lenguaje, por lo que debe tener su reflejo también en ELE. No obstante, la realidad es que, pese a que han existido intentos brillantes por reelaborar el concepto de cultura y su didáctica en $\mathrm{ELE}^{3}$, aún conservamos la cultura como un apéndice, un recurso con el que salpimentar la docencia. En las siguientes líneas trataremos de ofrecer una alternativa para integrar la cultura en la práctica docente.

\section{LENGUA Y CULTURA: EL CASO EN ELE}

La asunción de que los contenidos culturales son necesarios para un buen desarrollo de la competencia comunicativa ha propiciado que estos se incorporen a los currículos de lenguas, ya que deben formar parte de la enseñanza real. Sin embargo, una de las principales dificultades a las que nos enfrentamos respecto al papel de la cultura en ELE es su propia conceptualización. Como vimos en el apartado anterior, el término es ambivalente y se hace necesario acotar los límites respecto a lo que es cultura y lo que no, o, simplemente, qué cultura se debe trasladar al aula.

Para ello, se impone partir de la reflexión sobre cómo debe ser la cultura que se incluya en la enseñanza de español como lengua extranjera. En nuestro caso, consideramos que lo más oportuno es partir de una cultura eminentemente comunicativa. Tradicionalmente, en nuestra disciplina se han diferenciado dos tipos de culturas: la gran cultura o 'highbrow culture', en la que se encuentran las artes, la política, la historia o la economía; y la cultura de superficie o 'lowbrow culture', donde se ubican las tradiciones o las normas de comportamiento (Bueno, 1995). Miquel y Sans (1992 [2004]) añaden un tercer tipo, la 'kultura con k' o 'dialectos culturales', es decir, variedades de un grupo social o de una zona concreta.

En nuestra opinión, estas concepciones culturales no cubren las necesidades del alumnado, además de generar una dificultad terminológica para clasificar diferentes hechos culturales: las costumbres que rodean al acto de la comida, -temas de los que se puede hablar y de los que no, orden de los platos, si se puede o no abandonar la mesa antes de que finalice el resto de comensales- en España, ¿forman parte de la 'highbrow culture' o, en términos de Miquel y Sans "cultura a secas"?, ¿se sitúan en la misma categoría que los patrones culturales que rigen un saludo o una despedida en España? El problema no es solo epistemológico, un ejercicio de reflexión teórica, sino que supone decidir qué contenidos deben figurar en el currículo, por qué y cómo se pueden llevar a cabo en la práctica docente.

\footnotetext{
${ }^{3}$ Para algunas propuestas para trabajar la cultura en ELE pueden consultarse, entre otros, Miquel y Sans (1992 [2004]), Carcedo González (1996), Oliveras (2000), Iglesias Casal (2003), Trujillo Sáez (2005) o Estévez Coto y Fernández de Valderrama (2006).
} 
Nuestra concepción cultural surge de la perspectiva que contempla los hechos culturales en una doble vertiente: aquellos que influyen directamente en la eficacia de la comunicación y los que no. El Grupo CRIT $(2003,2006)$ denomina a la primera categoría 'cultura comunicativa' y a la segunda 'cultura material'. Según ellos, la cultura comunicativa

se manifiesta básicamente en las formas de conversar, en las formas de interactuar. Los individuos de cada cultura, [...] tienen su forma característica de gestualizar, de intercambiar los turnos de palabra, de organizar las conversaciones, de dirigirse a sus interlocutores, de abordar los temas delicados, etc. (Grupo CRIT 2006: 26).

Consideramos que la cultura comunicativa es un buen punto de partida para organizar los contenidos culturales por tres razones: es necesaria, puesto que depende de procesos cognitivos inconscientes y ligados a la cultura de origen; es rentable, es decir, su conocimiento redunda en beneficio de los propios estudiantes, y es integradora, ya que está relacionada con el resto de las competencias. Veamos cada uno de ellos.

\subsection{La necesidad de incluir la cultura comunicativa en la práctica docente}

El concepto de cultura es tan amplio que, según el enfoque del que partamos, incluirá unas realidades u otras. Por esta razón, se hace necesario concebir la cultura desde una perspectiva que esté vinculada con la enseñanza de lenguas, y más en concreto, con la eficacia comunicativa. Geertz, padre de la antropología simbólica, considera la cultura como un conjunto de símbolos mediante los que la sociedad se comunica:

El concepto de cultura que propugno [...] es esencialmente un concepto semiótico. Creyendo con Max Weber que el hombre es un animal inserto en tramas de significación que él mismo ha tejido, considero que la cultura es esa urdimbre y que el análisis de la cultura ha de ser por lo tanto, no una ciencia experimental en busca de leyes, sino una ciencia interpretativa en busca de significaciones (Geertz 1973 [1992]: 20).

De esta manera, la cultura se concibe como un ejercicio de interpretación que es posible en una comunidad gracias a que sus miembros conocen los modos de codificar y descodificar los símbolos que se manifiestan. Asimismo, aquellas personas ajenas a este grupo y que, por lo tanto, desconozcan las pautas culturales, corren el peligro de malinterpretar un hecho cultural.

$\mathrm{Si}$ aceptamos esta concepción, debemos plantearnos cómo es el proceso interpretativo que hace posible la significación. Para ello, recurrimos a los presupuestos del interaccionismo simbólico ${ }^{4}$, según los cuales la base de la sociedad es la comunicación. Según ellos, es imposible no comunicar, puesto que toda acción -aunque no sea verbal- significa algo para el resto y es a través de la comunicación cómo se negocian los significados y la conducta humana. Esto quiere decir que pese a haber unos patrones de comportamiento generalmente estables, la conducta de los miembros de la sociedad es en cierta medida creativa, ya que se adecua a las respuestas

\footnotetext{
${ }^{4}$ La corriente surge a través de las propuestas de George H. Mead. Póstumamente se publicaron sus apuntes (Mead, 1962 [1999]).
} 
que se reciben del resto de las personas durante la interacción (Rodrigo Alsina, 1999: 177).

En conclusión, afrontar la cultura en la enseñanza de idiomas desde una perspectiva semiótica e interactiva nos permite vincular estos contenidos con la comunicación. De hecho, el conocimiento cultural es necesario en la didáctica delenguas extranjeras por dos motivos: en primer lugar, porque permite atribuir significados a las conductas de los hablantes $y$, por otra parte, porque ayuda a establecer esquemas previsibles respecto a la conducta del resto. En cuanto a la cultura como herramienta predictiva, Goodenough considera que

Una descripción válida de una cultura como algo aprendido es la que predice si una acción particular será o no aceptada por aquellos que conocen la cultura como conforme a sus normas de conducta (Goodenough, 1971: 194).

Estas predicciones que se realizan a través del conocimiento cultural tienen una doble utilidad. Por una parte, sirven para prever la conducta aceptada por una comunidad $\mathrm{y}$, por otra, hacen posible que se prediga si un comportamiento propio será adecuado o no según las normas establecidas para un determinado contexto. Este segundo uso "permite que la gente estreche el campo de variaciones probables a unas pocas alternativas" (Goodenough, 1971: 194).

El último aspecto que hemos de tratar para definir nuestro objeto de estudio es la variedad de los hechos culturales. Por naturaleza, la cultura influye en múltiples realidades de un grupo social, por lo que hemos de decidir qué contenidos de la cultura son pertinentes en la enseñanza de español como lengua extranjera. Como avanzamos en el apartado anterior, creemos que la diferenciación entre 'cultura material' y 'cultura comunicativa' es muy esclarecedora para la didáctica de lenguas:

(1) La gestión del tiempo en España: puntualidad en las citas.

(2) Un apretón de manos.

(3) Manueeeeel.

(4) El sistema educativo español.

(5) Baja la música.

(6) Costumbres españolas: ir de tapas.

(7) ¿Qué tal la mujer? ¿Y los hijos?

(8) Mantener/desviar la mirada.

Desde la óptica de la antropología semiótica, todos estos hechos culturales tienen un significado en nuestra cultura. Ante una cita, que uno de los participantes llegue con diez minutos de retraso será interpretado por una persona española de manera diferente a como, con toda seguridad, lo interpretará una anglosajona, debido a, como hemos visto, las diferencias respecto a las culturas de origen de ambos. Si esa situación se estuviera produciendo en España, el suceso puede dar lugar a un malentendido por parte del anglosajón al atribuir un significado a la conducta del español que este no comparte. 
Asimismo, podemos observar en este mismo caso que ese hecho cultural se pone en juego a través de la interacción entre los miembros de una comunidad. Gracias a este proceso se pueden atribuir significados a la conducta de las otras personas y también predecirlas, tanto a nivel grupal -para conocer los patrones de una comunidadcomo a nivel individual - para saber si un comportamiento será aceptado o no. En el caso anterior, el anglosajón puede predecir, a través de varias interacciones, que en la cultura española está comúnmente aceptado llegar con un pequeño retraso a una cita y puede, por lo tanto, decidir actuar en consecuencia. Sin embargo, la predicción individual depende también del contexto: si en lugar de un encuentro con un amigo para una reunión distendida nos encontramos ante una cita para acudir a un evento con horario fijado - por ejemplo, una función teatral-, el anglosajón debe saber que en ese contexto su compañero valorará positivamente la puntualidad. Es decir, los patrones culturales son estables pero se negocian a través de la interacción.

Podríamos analizar del mismo modo como hemos hecho con (1) el resto de los hechos culturales. Sin embargo, entre ellos existe una diferencia: (1), (4) y (6) no están íntimamente relacionados con el éxito en la comunicación, a no ser que el tema de la misma gire en torno a uno de estos contenidos culturales. Es más, un foráneo que conozca estos hechos culturales pero que no tenga ningún conocimiento ni de la lengua ni de la cultura comunicativa española puede interactuar adecuadamente respecto a (1), (4) y (6), siempre y cuando se use un código lingüístico que conozca, disponga de una buena competencia estratégica o, en su defecto, no se use ninguno.

No sucede lo mismo con el resto de los ejemplos: un extranjero debe conocer en qué situaciones puede iniciar una conversación con (2) y en cuáles no, incluso diferenciarlo de su significado en una negociación; las connotaciones que tiene en español pronunciar (3) al saludar a una persona; en qué situaciones una orden como (5) es adecuada y cuándo debe optar por un acto de habla indirecto; en qué registros puede preguntar por información personal (7) y cuáles son las respuestas prototípicas a estas preguntas o qué implicaciones tiene que un interlocutor desvíe o mantenga su mirada en él.

Todos son hechos simbólicos de nuestra cultura $\mathrm{y}$, como tales, tienen un significado; sin embargo, solo depende de algunos de ellos que la comunicación se desarrolle dentro de los parámetros adecuados. Como veremos más adelante, (1), (4) y (6) pertenecen a los 'referentes culturales' o 'saberes y comportamientos socioculturales' (Instituto Cervantes, 2006) mientras que (2), (3), (5), (7) y (8) son 'patrones comunicativos' (Grupo CRIT, 2003, 2006; Raga Gimeno, 2005).

En conclusión, la cultura comunicativa debe formar parte activa de la práctica docente de ELE porque:

- Es interpretativa: por su naturaleza semiótica, el estudiante debe aprender a asignar los significados de la cultura meta y no de su cultura de origen para evitar malentendidos.

- Es creativa: se pone de manifiesto en la interacción y a través de esta se negocia la conducta, por lo que el seguimiento de los patrones comunicativos 
depende del contexto. Los alumnos necesitan conocer esta variabilidad y desarrollar estrategias predictivas.

- Es comunicativa: el conocimiento lingüístico no es suficiente para asegurar una comunicación eficaz.

\subsection{La rentabilidad de la cultura comunicativa en la enseñanza de lenguas}

Como docentes de una lengua extranjera hemos de suponer que los estudiantes van a desenvolverse, más pronto que tarde, en contextos interculturales. Dejando a un lado la barrera que puede suponer el desconocimiento del código lingüístico, en estas situaciones se generan un conjunto de procesos afectivos, psicológicos e incluso cognitivos que pueden dificultar el desarrollo de la interacción. En numerosas ocasiones, estos factores "externos" al lenguaje desembocan en una comunicación ineficaz.

Uno de los conflictos a los que se enfrenta un hablante al integrarse en una comunidad distinta a la suya es el choque cultural, el cual "is precipitated by the anxiety that results from losing all our familiar signs and symbols of social intercourse" (Oberg, 1960: 177). La ansiedad de la que habla Oberg se produce tanto por la ausencia de referentes culturales como por la falta de reconocimiento de los patrones comunicativos de esa comunidad. Es decir, el conflicto no surge exclusivamente porque las festividades, las tradiciones o la arquitectura de esa cultura son diferentes a las suyas sino que también -y de manera más trascendental- se debe a que las pautas que rigen la interacción comunicativa no significan lo mismo que en su cultura de origen.

Dos factores decisivos en la comunicación intercultural son la ansiedad y la incertidumbre. Gudykunst $(1987,2005)$ observa que estos procesos psicológicos repercuten negativamente en la comunicación y se plantea cómo podemos gestionarlos para conseguir una comunicación efectiva, la cual

no significa totalmente controlada y sin ambigüedades. Una comunicación efectiva es aquella que llega a un grado de comprensión aceptable por parte de los interlocutores. No se trata de buscar una comunicación perfecta, sino efectiva (Rodrigo Alsina, 1999: 168).

La incertidumbre se produce cuando un forastero - una persona atribuida a un grupo diferente al de referencia- entra en contacto con un hablante de la cultura meta. Puede ser de dos tipos, predictiva y explicativa: la primera se refiere a la inseguridad respecto a la predicción de las conductas, creencias o sentimientos del otro que tienen lugar durante la interacción; la explicativa está relacionada con las dificultades que tiene el forastero para comprender determinadas reacciones, puesto que las interpreta en base a sus patrones culturales. Según Gudykunst, el nivel de incertidumbre debe mantenerse en un punto que motive al foráneo a conocer la cultura meta sin que provoque ansiedad en sus interacciones, ya que cuando la ansiedad es alta recurrimos a los prototipos para interpretar la interacción y, generalmente, produce una comunicación ineficaz. Por lo tanto, a mayor conocimiento de la cultura comunicativa de acogida, menor incertidumbre, menor ansiedad y mayor motivación. 
Estrechamente relacionada tanto con la gestión de la ansiedad y de la incertidumbre como con la cultura comunicativa está la 'host communication competence' (Kim, 1988; 1995). Esta es la capacidad que tiene el hablante para codificar y descodificar información de acuerdo a los patrones culturales de la comunidad de acogida. El desarrollo de la 'host communicative competence' depende de aspectos afectivos y psicológicos, del conocimiento del sistema de la cultura de acogida y de la capacidad para actuar en las interacciones de acuerdo a las pautas de esa comunidad. Según Kim, cuando un foráneo ha adquirido suficiente nivel en esta competencia se muestra más seguro y menos dependiente de los miembros de esa comunidad para sus interacciones.

Del mismo modo, es necesario incluir contenidos culturales en la práctica docente para evitar actitudes de minorización (CRIT, 2006) entre nuestros estudiantes. Cuando un hablante se enfrenta a una situación en la que la comunicación es complicada, es inevitable que tienda a valorar negativamente la cultura del otro interlocutor. Esto se debe a que se juzga el comportamiento del otro como apropiado o inapropiado desde nuestra perspectiva cultural. Ante esta situación, Byram y Fleming recuerdan la responsabilidad del docente y del alumno de lenguas extranjeras de aprender a ser hablantes interculturales.

El 'hablante intercultural' es una persona que tiene conocimientos de una, o preferentemente de más culturas e identidades sociales y que disfruta de la capacidad de descubrir y de relacionarse con gente nueva de otros entornos para los que no ha sido formado de forma intencional (Byram y Fleming, 1998 [2001]: 16).

El hablante intercultural difiere del perfecto hablante 'lingüístico' en que este último busca llegar al nivel nativo para que se le pueda confundir con uno de ellos. Sin embargo, el hablante intercultural reconoce su cultura y la del interlocutor y es capaz de mediar entre ellas, explicando las diferencias y aceptándolas. Las actitudes de minorización, por lo tanto, se pueden evitar, o al menos minimizar, si integramos la cultura comunicativa en ELE.

Por último, hemos de considerar dos aspectos que están íntimamente ligados con la práctica docente. En primer lugar, cuando se incluyan contenidos culturales se debe ser precavido con el objetivo que se persigue:

Los estudiantes deberán desarrollar la competencia pragmalingüística propia de los hablantes nativos, esto es, asignar a las estructuras lingüísticas la misma fuerza pragmática que los hablantes nativos, pero deberán tener la libertad de adoptar la competencia sociopragmática del hablante nativo, esto es, compartir los juicios de valor de un hablante nativo (Kramsch, 1998 [2001]: 31).

Es decir, la razón por la que se incluyen los contenidos culturales es para que se pueda interpretar la comunicación -verbal y no verbal- en los mismos parámetros que los hablantes nativos con el fin de que la comunicación sea más efectiva, no para que los estudiantes valoren los hechos culturales de la misma manera que la comunidad de la cultura meta. Ambas posturas no son excluyentes y están íntimamente ligadas a los conceptos de 'hablante intercultural' y 'consciencia intercultural'. Respecto a la consciencia intercultural, el MCERL dice: 
El conocimiento, la percepción y la comprensión de la relación entre el «mundo de origen» y el «mundo de la comunidad objeto de estudio» (similitudes y diferencias distintivas) producen una consciencia intercultural, que incluye, naturalmente, la conciencia de la diversidad regional y social en ambos mundos, que se enriquece con la conciencia de una serie de culturas más amplia de la que conlleva la lengua materna y la segunda lengua, lo cual contribuye a ubicar ambas en su contexto (Consejo de Europa, 2002: 101).

Ante un acto de habla directivo como (5) 'baja la música' en el que la orden se expresa mediante el uso del imperativo, un estudiante necesita saber -tanto como para producirlo como para interpretarlo- la fuerza ilocucionaria de ese enunciado y el significado implícito que conlleva en la cultura comunicativa española dependiendo del contexto de aparición. Sin embargo, es libre de valorarlo como quiera -por ejemplo, negativamente porque lo considera brusco, inadecuado o descortés-, aunque este hecho dependerá de su sensibilidad o consciencia intercultural.

Debemos abrir, en este momento, un paréntesis aclaratorio: entendemos por valorar un hechocultural como el proceso consciente y deliberado por el cual un hablante emite un juicio respecto a una -o parte de una-cultura. No se debe confundir con los procesos afectivos que surgen en una persona cuando se enfrenta a un hecho cultural de una comunidad ajena a la suya. Estos procesos son inconscientes, involuntarios y afectan a la comunicación intercultural, de ahí la necesidad de trabajarlos de manera explícita en el aula. En el caso anterior, es probable que un miembro de una "cultura comunicativa distante" (Grupo CRIT, 2003, 2006; Raga Gimeno, 2005) o "cultura de distanciamiento" (Briz Gómez, 2007) sienta esa emisión como una intromisión en su espacio personal o un fenómeno descortés.

El segundo aspecto conectado con la práctica docente tiene que ver con el profesorado. Como vimos en el apartado anterior, la cultura comunicativa está ligada a una serie de procesos interpretativos que dependen de la cultura de origen de la persona. Hoy en día, el español se enseña en 93 países que no tienen el español como lengua oficial (Instituto Cervantes, 2014: 11), por lo que podemos suponer que un gran número de docentes pertenecen a culturas de origen diferentes a la española ${ }^{5}$. Para este profesorado, es especialmente útil que el currículo de ELE recoja y sistematice los contenidos comunicativos culturales.

Como vemos, muchas de las dificultades en la comunicación intercultural provienen de la impronta que deja la cultura de una comunidad en los modos de significar. Según lo expuesto en este apartado, podemos concluir que la integración de la cultura comunicativa en ELE es rentable porque:

- Disminuye el choque cultural: el trabajo cultural previo a la inmersión lingüística favorece el reconocimiento de los patrones comunicativos de la cultura meta.

- Reduce aspectos psicológicos como la ansiedad y la incertidumbre: genera estrategias de predicción de conducta de la comunidad objeto de estudio.

\footnotetext{
${ }^{5}$ Un tema tangencial al objetivo de este trabajo, y en el que no podemos entrar por motivos de espacio, es la conveniencia o no de enseñar una variedad cultural panhispánica desde esta perspectiva.
} 
- Aumenta la confianza de hablante: la cultura comunicativa colabora con el desarrollo de la 'host communicative competence'.

- Rebaja las actitudes de minorización.

- Repercute en la comunicación eficaz: favorece el desarrollo de la competencia pragmalingüística.

- Sirve de referencia para el profesorado no nativo.

\subsection{Bases para integrar el componente cultural en el currículo de ELE}

Una vez planteada la naturaleza de la cultura comunicativa y las ventajas que genera trasladarla a la práctica docente, debemos esclarecer cómo podemos integrarla en el currículo de ELE. Para ello, estos contenidos deben cumplir con dos características: reflejar interdependencia con la competencia comunicativa, es decir, que un mayor desarrollo de la competencia cultural suponga un perfeccionamiento de la competencia comunicativa del hablante $y$, por otra parte, poder ser nivelados.

\subsubsection{El vínculo entre el interaccionismo simbólico y la competencia comunicativa}

Según el MCERL la competencia comunicativa está compuesta por los subcomponentes lingüístico, sociolingüístico y pragmático, cada uno de los cuales engloba destrezas, habilidades y conocimientos (Consejo de Europa, 2002: 13). Como puede observarse, no explicita la existencia de un componente cultural aunque a lo largo del texto se mencionen las destrezas interculturales, la consciencia intercultural o la competencia pluricultural. En nuestra opinión, esto se debe a que los contenidos culturales tienen un campo de influencia 'pluricomponencial', lo que dificulta dotarlos de una identidad propia. Desarrollar contenidos culturales desde la óptica del interaccionismo simbólico supone trabajar la interculturalidad; la competencia estratégica y la acción comunicativa, es decir, la pragmática.

Canale y Swain (1980), Canale (1983 [1995]), Bachman (1990), Celce-Murcia, Dörnyei y Turrell (1995) ya incluyen la competencia estratégica en sus modelos de competencia comunicativa. Esta competencia, a menudo denostada en el campo de ELE (Vázquez, 2012), se entiende como la capacidad del hablante para relacionar el conocimiento lingüístico con las estructuras cognitivas propias y el contexto comunicativo. Cuando incluimos contenidos culturales comunicativos estamos dotando a los estudiantes de herramientas para que consigan una comunicación más efectiva en sus interacciones, máxime cuando estas se producen en situaciones de interculturalidad. Estévez Coto y Fernández de Valderrama (2006: 23) consideran que la adquisición de la competencia comunicativa "ha de ir unida a la adquisición de una competencia social que posibilite una integración intercultural del estudiante" y el PCIC plantea entre sus objetivos que el alumno

llegue a convertirse en intermediario cultural mediante la activación de determinadas estrategias y la capacidad de afrontar situaciones de malentendidos y conflictos de carácter cultural (Instituto Cervantes, 2006: 76, vol. I). 
Sin embargo, consideramos que es en la pragmática donde la cultura comunicativa tiene mayor relevancia. Hernández Sacristán (1999), a propósito de la pragmática intercultural, describe esta relación de la siguiente manera:

Cabe ahora plantearse el tema de si la imbricación entre fenómenos pragmáticos y culturales (entiéndase no específicamente lingüísticos) se resuelve sencillamente en el hecho de que toda categoría pragmática es, por su propia naturaleza, una realidad bifronte (lingüístico-cultural), o si, dando esto último por descontado, existirían también relaciones globales entre las características propias de un código pragmático y las de un entorno sociocultural (Hernández Sacristán, 1999: 27).

Es decir, la cultura, en cuanto conjunto de conocimientos necesarios para llevar a cabo una interacción comunicativa de acuerdo a los patrones de actuación de una comunidad, está indisociablemente ligada a las estructuras y estrategias pragmáticas que se ponen en uso en cada situación. A este respecto, Cenoz (2000) apunta que algunos investigadores conciben dos dimensiones en la subcompetencia pragmática: la pragmalingüística y la sociopragmática o filtro cultural. Mientras que la primera se refiere a las estructuras y los exponentes lingüísticos utilizados en los diferentes actos de habla, la segunda

se ocupa de aquellos aspectos del uso y la interpretación de las formas lingüísticas que están determinados social y culturalmente, tratando de identificar cuáles son las normas que regulan el comportamiento lingüístico (Escandell Vidal, 2006: 239).

Podemos considerar, entonces, que nuestra percepción del componente cultural se integra dentro del subcomponente pragmático, más en concreto, en la dimensión sociopragmática. Eso sí, no debemos obviar la repercusión que este componente tiene en la competencia estratégica e intercultural.

\subsubsection{La presencia de la cultura en el PCIC}

Una de las principales dificultades a las que nos enfrentamos cuando queremos incluir contenidos culturales en la didáctica de lenguas extranjeras es el de su nivelación y caracterización ya que, como vimos en el apartado 2.1., la cultura, en un sentido laxo, puede abarcar todas las realidades de una sociedad. En el PCIC encontramos contenidos culturales en los siguientes apartados:

- ‘6. Tácticas y estrategias pragmáticas'6.

- '10. Referentes culturales'.

- '11. Saberes y comportamientos socioculturales'.

- '12. Habilidades y actitudes interculturales'7.

\footnotetext{
${ }^{6}$ Aunque el propio PCIC no lo considera como uno de los pertenecientes al componente cultural, en la introducción a este apartado afirma que "el análisis en el aula va más allá de las cuestiones lingüísticas recogidas en este inventario" y remite a '11. Saberes y comportamientos socioculturales'.

${ }^{7} \mathrm{Si}$ consideramos la relación que se establece entre el subcomponente cultural y el desarrollo del componente estratégico -al menos en cuanto a las estrategias de expresión, comprensión, interacción y mediación- (Consejo de Europa, 2002: 60-87) deberíamos incluir también el apartado '13. Procedimientos de aprendizaje' del PCIC.
} 
A priori, parece que lo más lógico es consignar todo el componente cultural en un solo apartado, como el resto de los componentes. Sin embargo, esta dispersión de los contenidos se debe al criterio de oponer 'lo lingüístico' a 'lo que tiene proyección comunicativa':

El desarrollo de la dimensión cultural en estos tres inventarios [referentes culturales, saberes y comportamientos socioculturales y habilidades y actitudes interculturales] incorpora el tratamiento de aspectos que, si bien no son estrictamente lingüísticos, tienen relación directa con la lengua en su proyección comunicativa. Estos aspectos permiten al alumno el acceso a una realidad nueva sobre una base amplia e integradora en la que se imbrican los conocimientos, las habilidades y las actitudes que conforman un modo de 'competencia intercultural' (Instituto Cervantes, 2006: 537, vol. III).

Es decir, por un lado la cultura se organizará y se nivelará de una forma diferente al resto de los componentes porque es un fenómeno no lingüístico, pero, por otra, debe figurar en el currículo de una lengua extranjera porque influye en la comunicación. Esta diferenciación producirá que la cultura quede desconectada del resto de los componentes. Veamos, brevemente, qué contenidos culturales incluyen cada uno de estos tres apartados.

'Habilidades y actitudes interculturales' es un repertorio de estrategias que permiten al alumno acercarse a otras culturas tanto para desenvolverse como para interpretar hechos de estas comunidades. Se trata de un inventario diferente al resto del PCIC puesto que está organizado en torno a cuatro apartados sin nivelación de ningún tipo. Es, por lo tanto, un modo de ser hablante intercultural y de afrontar la interculturalidad que el docente debe fomentar en los estudiantes. En este apartado no se trata el hecho cultural en sí sino los modos - generales, no específicos- que permiten el acercamiento a estas realidades.

'Referentes culturales' recoge unidades de la cultura con ' $C$ ' o 'highbrow culture', es decir, se trata de un conocimiento de tipo factual, enciclopédico y acumulativo que permite al estudiante comprender la realidad del mundo hispanohablante: geografía, educación, sanidad, personajes históricos, arte, música, etc. Es decir, engloba la cultura material.

Los 'saberes y comportamientos socioculturales' dan cuenta de la identidad colectiva, la organización social, las relaciones personales, etc. de España, no de todo el mundo hispanohablante ${ }^{8}$. Los saberes forman parte del conocimiento declarativo y recogen aspectos relacionadas con las costumbres o las formas de organización de la sociedad: cuáles son las partes de la comida o del día, por ejemplo. Los comportamientos, sin embargo, son cuestiones que se ponen de manifiesto a través de la interacción: la puntualidad, la vestimenta o la comunicación no verbal asociada a diferentes situaciones. En este apartado se remite frecuentemente a 'Tácticas y estrategias pragmáticas'. Según el PCIC,

Todos estos conocimientos - en forma de saberes y comportamientos - son imprescindibles si se desea una comunicación rentable y eficaz. Al igual que sucede con los referentes culturales, el

\footnotetext{
${ }^{8}$ Según el PCIC, esto se debe a la gran variabilidad de estas unidades en las sociedades del mundo hispanohablante (véase nota 5).
} 
alumno debe ser capaz de poner en relación sus competencias lingüísticas con el conocimiento de aspectos socioculturales que van a determinar la elección de los exponentes lingüísticos, del vocabulario, del registro, que deberá utilizar según la situación comunicativa en que se encuentre. Por ejemplo, para dirigirse a un desconocido, el alumno no solo deberá ser capaz de hacer uso de las funciones lingüísticas y el vocabulario adecuados, sino que también deberá ser muy consciente de los factores extralingüísticos (uso del espacio, del tiempo, de los movimientos corporales) que se dan en esa situación comunicativa y cuyo desconocimiento puede ser fatal para el éxito de la comunicación (Instituto Cervantes, 2006: 573, vol. III).

Observamos dificultades para conceptualizar qué tipo de cultura se recoge en este apartado. La mayoría de los saberes y comportamientos podrían caracterizarse como 'lowbrow culture' -aunque hay algunos casos fronterizos como las festividades o la gastronomía local- pero tendríamos más dificultades para considerar estas unidades como 'cultura material' o 'cultura comunicativa'. Más complicado aún es decidir si son "estrictamente lingüísticos" o "tienen proyección comunicativa” (Instituto Cervantes, 2006: 537, vol. III).

Consideramos que la distinción entre 'lo que es lingüístico' y 'lo que tiene proyección comunicativa' es muy confusa. De hecho, prueba de ello es la segregación de los contenidos culturales en el $P C I C$, aspecto que repercute negativamente en la práctica docente puesto que no quedan claros los límites sobre qué cultura debemos llevar al aula y en qué momento. La siguiente tabla recoge, a modo de muestra, algunos hechos culturales extraídos del PCIC de los $\operatorname{apartados}^{9}$ 6, 10 y 11 y su naturaleza atendiendo a la oposición 'lingüístico/proyección comunicativa'; 'highbrow culture' /'lowbrow culture' y 'cultura material' /'cultura comunicativa':

\begin{tabular}{|l|c|c|c|c|}
\hline \multicolumn{1}{|c|}{ Hecho cultural } & $\begin{array}{c}\text { Apartado } \\
\text { PCIC }\end{array}$ & $\begin{array}{c}\text { Lingüístico } \\
\text { / Proyección } \\
\text { comunicativa }\end{array}$ & $\begin{array}{c}\text { Highbrow / } \\
\text { Lowbrow }\end{array}$ & $\begin{array}{c}\text { Material / } \\
\text { Comunicativo }\end{array}$ \\
\hline Actos de habla indirectos & 6. & Lingüístico & ----- & Comunicativo \\
\hline Halagos, cumplidos y piropos & 6. & Lingüístico & ----- & Comunicativo \\
\hline $\begin{array}{l}\text { Atenuación del acto } \\
\text { amenazador }\end{array}$ & 6. & Lingüístico & ---- & Comunicativo \\
\hline Pausas con valor modal & 6. & $\begin{array}{c}\text { Proyección } \\
\text { comunicativa }\end{array}$ & ----- & Comunicativo \\
\hline Alargamientos fónicos & 6. & Lingüístico & ----- & Comunicativo \\
\hline Canales de televisión & 10. & ----- & Lowbrow & Material \\
\hline $\begin{array}{l}\text { Premios Nobel en los países } \\
\text { hispanos }\end{array}$ & 10. & ----- & Highbrow & Material \\
\hline La guerra civil española & 10. & ----- & Highbrow & Material \\
\hline Religiones mayoritarias & 10. & ----- & Highbrow & Material \\
\hline Redes de transporte urbano & 10. & & Lowbrow & Material \\
\hline $\begin{array}{l}\text { Productos típicos por zonas o } \\
\text { regiones }\end{array}$ & 11. & ----- & Highbrow & Material \\
\hline
\end{tabular}

${ }^{9}$ Excluimos el apartado 13 por no presentar hechos culturales, sino habilidades y actitudes para afrontar estos hechos. 


\begin{tabular}{|c|c|c|c|c|}
\hline Hecho cultural & $\begin{array}{l}\text { Apartado } \\
\text { PCIC }\end{array}$ & $\begin{array}{l}\text { Lingüístico } \\
\text { / Proyección } \\
\text { comunicativa }\end{array}$ & $\begin{array}{l}\text { Highbrow / } \\
\text { Lowbrow }\end{array}$ & $\begin{array}{l}\text { Material / } \\
\text { Comunicativo }\end{array}$ \\
\hline $\begin{array}{l}\text { Frases y fórmulas utilizadas en } \\
\text { la mesa y momento en el que se } \\
\text { dicen }\end{array}$ & 11. & $\begin{array}{c}\text { ¿Lingüístico? } \\
\text { ¿Proyección } \\
\text { comunicativa? }\end{array}$ & Lowbrow & Comunicativo \\
\hline $\begin{array}{l}\text { Convención social respecto al } \\
\text { regateo }\end{array}$ & 11. & ----- & Lowbrow & Material \\
\hline \multirow{2}{*}{ 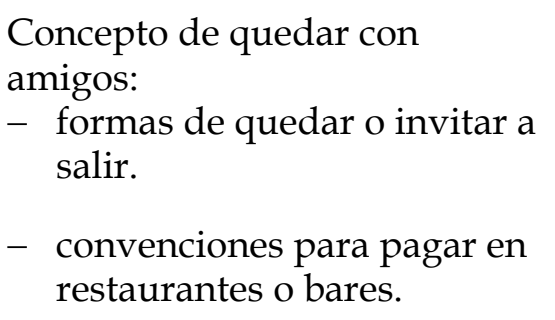 } & \multirow{2}{*}{11.} & Lingüístico & Lowbrow & Comunicativo \\
\hline & & ---- & Lowbrow & Material \\
\hline $\begin{array}{l}\text { Usos sociales del beso y del } \\
\text { abrazo }\end{array}$ & 11. & $\begin{array}{l}\text { Proyección } \\
\text { comunicativa }\end{array}$ & Lowbrow & Comunicativo \\
\hline
\end{tabular}

Tabla 1. Hechos culturales clasificados según diferentes teorías.

Como puede observarse, la caracterización ofrecida por el PCIC es poco clara. Por un lado, para los hechos culturales de conocimiento factual la dicotomía 'lingüístico/proyección comunicativa' no es significativa, a no ser que se considere que todo aquello sobre lo que se puede entablar una conversación tiene proyección comunicativa, lo cual no sería rentable en un currículo debido a que las variables son infinitas. Como vimos, son conocimientos acumulativos que cualquier persona puede poseer, con independencia de que conozca la lengua o no.

Por otro lado, las unidades que se realizan a través de las estructuras de la lengua -actos de habla indirectos, halagos, cumplidos y piropos, etc.- tienen, como no podía ser de otra manera, proyección comunicativa. Asimismo, pese a que formalmente correspondan al plano de lo lingüístico, su eficacia no solo depende de la corrección lingüística sino también de la adecuación a la situación la cual, a su vez, varía según la cultura en la que se realice la interacción (González Plasencia, en prensa). En resumen, son unidades que no se pueden desvincular del componente cultural.

Finalmente, hemos de destacar la inestabilidad de la estructuración del PCIC en cuanto a los contenidos culturales. Como vemos en la tabla, en los apartados 6 , 10 y 11 se mezclan tanto hechos 'lingüísticos' como 'de proyección comunicativa' e incluso figuran otros para los que este binomio no es pertinente. La clasificación tampoco responde a la distinción clásica entre 'highbrow culture' y 'lowbrow culture' puesto que en el apartado 10 y 11 se mezclan realidades de ambas esferas. Además, esta caracterización no considera las emisiones lingüísticas que dependen de la cultura -'tácticas y estrategias pragmáticas'.

Consideramos que la conceptualización más conveniente para organizar los contenidos culturales es la que diferencia entre las realidades materiales y las comunicativas. Como se observa en la tabla, permite clasificar todas las unidades en 
torno a un criterio estable, aspecto que redunda en la práctica docente. Sin embargo, hemos de señalar que esta caracterización tampoco se mantiene estable a lo largo de los apartados, hecho que se debe a que no se ha dotado al componente cultural de una identidad propia.

\subsubsection{La nivelación de la cultura en el PCIC}

Si anteriormente analizábamos la organización de los contenidos culturales en el PCIC, en este punto comentaremos la nivelación de los mismos. Hemos de comenzar destacando que el PCIC propone una progresión no por niveles sino por fases (aproximación, profundización y consolidación) para los 'Referentes culturales' y para los 'Saberes y comportamientos socioculturales' ${ }^{10}$, mientras que las 'Habilidades y actitudes interculturales' las desarrolla a modo de inventario sin ningún tipo de gradación. El PCIC aduce los siguientes motivos:

- La enseñanza de estos contenidos depende de variables idiosincrásicas o de aspectos específicos de cada situación de enseñanza que solo pueden ser ponderados a través de un análisis detallado de la situación de enseñanza (Instituto Cervantes, 2006: 42, vol. I).

- En el MCERL no hay escalas descriptivas para el componente cultural ni para las competencias generales (Instituto Cervantes, 2006: 41, vol. I).

- La organización modular de los contenidos posibilita distintas aplicaciones según considere el docente qué es lo más apropiado (Instituto Cervantes, 2006: 450 , vol. I).

- El desarrollo de la competencia comunicativa (subcompetencias estrictamente lingüísticas) y la competencia cultural no tienen por qué ser parejos (Instituto Cervantes, 2006: 449, vol. I).

Veamos, ahora, los criterios internos a cada apartado para nivelar los contenidos. Como ya hemos comentado anteriormente, 'Habilidades y actitudes interculturales' no presenta hechos culturales sino estrategias para acercarse a la cultura meta y desarrollar la interculturalidad, motivo por el cual no podemos profundizar más en su análisis. Respecto a la gradación de los 'Referentes culturales', el PCIC dice:

Es importante tener en cuenta que estas fases [aproximación, profundización y consolidación] no tienen una correspondencia sistemática con los niveles de gradación establecidos en la dimensión lingüística del inventario. Los criterios de gradación de los contenidos del inventario de Referentes culturales han sido, básicamente, el mayor o menor grado de universalidad y el mayor o menor grado de accesibilidad (Instituto Cervantes, 2006: 539, vol. III).

Se entiende que un referente cultural es 'más universal' cuanta más repercusión tiene fuera del mundo hispanohablante y, por lo tanto, es más probable que los estudiantes lo conozcan o puedan establecer relaciones con otros hechos culturales. Por otra parte, la 'accesibilidad' de los referentes culturales depende de la frecuencia de aparición que tengan en el mundo hispanohablante y en el resto de países además

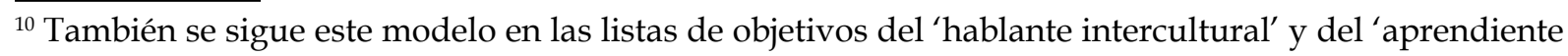
autónomo'.
} 
de las facilidades de las que disponga el estudiante para acceder a ellos a través de otras fuentes.

En cuanto a la distribución por niveles de los 'Saberes y comportamientos socioculturales',

El criterio principal que se ha seguido para establecer la distribución en las tres fases es el de que los contenidos resulten más o menos necesarios para los contactos y las interacciones de los alumnos. Así, en el nivel de aproximación se presentan los contenidos relacionados con temas más cercanos a sus experiencias cotidianas. En los niveles de profundización y de consolidación los contenidos que se presentan responden a un análisis más sistemático de las estructuras sociales y de los fenómenos culturales, que permite ampliar las experiencias socioculturales inmediatas de los aprendientes (Instituto Cervantes, 2006: 574, vol. III).

Es decir, aquello que el hablante deba conocer para llevar a cabo de manera satisfactoria una interacción forma parte de la fase de aproximación mientras que las cuestiones que necesitan de una mayor profundización o que se deban a implicaciones interculturales se reservan para las otras dos fases.

En la siguiente tabla se resumen los apartados del PCIC donde aparecen hechos culturales, cómo ha sido nivelado cada apartado y qué criterio se ha seguido para ello.

\begin{tabular}{|c|c|c|c|}
\hline Apartado & Nivelación & \multicolumn{2}{|c|}{ Criterio(s) } \\
\hline $\begin{array}{l}\text { 6. Tácticas y estrategias } \\
\text { pragmáticas }^{11}\end{array}$ & $\begin{array}{l}\text { Niveles de referencia } \\
(\mathrm{A} 1, \mathrm{~A} 2, \mathrm{~B} 1, \mathrm{~B} 2, \mathrm{C} 1, \mathrm{C} 2)\end{array}$ & \multicolumn{2}{|c|}{$\begin{array}{l}\text { Progresión del alumno según el } \\
M C E R \text {, Nivel umbral, etc. }\end{array}$} \\
\hline \multirow{3}{*}{ 10. Referentes culturales } & Aproximación & + Universal & + Accesible \\
\hline & Profundización & Universal & Accesible \\
\hline & Consolidación & - Universal & - Accesible \\
\hline \multirow{3}{*}{$\begin{array}{l}\text { 11. Saberes y } \\
\text { comportamientos } \\
\text { socioculturales }\end{array}$} & Aproximación & \multicolumn{2}{|c|}{ + Necesario en la interacción } \\
\hline & Profundización & \multicolumn{2}{|c|}{ - Necesario en la interacción } \\
\hline & Consolidación & \multicolumn{2}{|c|}{ - Necesario en la interacción } \\
\hline $\begin{array}{l}\text { 12. Habilidades y actitudes } \\
\text { interculturales }\end{array}$ & Inventario único & \multicolumn{2}{|c|}{$\begin{array}{c}\text { Criterio del profesor según las } \\
\text { necesidades }\end{array}$} \\
\hline
\end{tabular}

Tabla 2. Nivelación de la cultura en el PCIC.

Como puede observarse, el PCIC utiliza hasta cinco criterios diferentes para nivelar los contenidos culturales - progresión del alumno justificada según repertorios anteriores, universalidad, accesibilidad, necesidad y criterio del profesor-, lo que resulta en tres tipos de distribución: por niveles de referencia, por fases o en un único inventario. Tanto la ausencia de un criterio único para nivelar el componente cultural como la dispersión del mismo en varios apartados dificultan la segunda concreción del currículo.

En nuestra opinión, la propuesta del PCIC para los 'Referentes culturales' es apropiada, ya que no hay relación entre la progresión de un estudiante en cuanto a su conocimiento factual y su competencia comunicativa. De este modo, ante referentes

\footnotetext{
${ }^{11}$ Insistimos, una vez más, en que el PCIC no considera este apartado dentro del componente cultural. Su inclusión se debe a la relación directa entre ambos y a las referencias continuas entre este y el resto de apartados culturales.
} 
culturales literarios como "Importancia de Cervantes y el Quijote en la historia de la literatura universal”, "Premios Nobel en los países hispanos: Juan Ramón Jiménez" y "Valle-Inclán y el esperpento" 12 -según el PCIC, fase de aproximación, profundización y consolidación, respectivamente- se hace difícil decidir qué debe saber un estudiante de A1 frente a otro de B2, puesto que en este plano lengua y cultura pertenecen a universos cognitivos diferentes.

En cuanto a las habilidades y actitudes interculturales recogidas en el apartado número 12 del PCIC, consideramos que su organización es adecuada a la naturaleza de las unidades expuestas, ya que son estrategias generales que permitan al estudiante acercarse al hecho cultural. Como sucede con los 'Referentes culturales', la sensibilidad intercultural de un estudiante no ha de estar ligada a su desarrollo lingüístico o comunicativo y solo el docente puede conocer los casos concretos. Sin embargo, hemos de destacar la escasa profundidad con la que se tratan algunas de estas habilidades, especialmente las relacionadas con la 'interacción cultural'13, puesto que estas influyen directamente en la eficacia comunicativa. Además, si el PCIC es una propuesta modular que pretende tender puentes entre los diferentes subcomponentes del lenguaje, echamos en falta referencias directas a otros apartados que vinculen 'lo estrictamente lingüístico' con 'lo que tiene proyección comunicativa', es decir, las habilidades que el estudiante debe adquirir para desenvolverse en interacciones interculturales.

Si bien consideramos, con matices, que la nivelación para los 'Referentes culturales' y las 'Habilidades y actitudes interculturales' es congruente, no podemos estar de acuerdo con la propuesta para los 'Saberes y comportamientos socioculturales' ya que, como vimos en el epígrafe anterior, en este apartado se mezcla la cultura material y la cultura comunicativa. Compartimos la idea de que la cultura material no puede organizarse según los niveles de referencia -de hecho, lo más oportuno es que estos contenidos se recojan en el apartado de 'Referentes culturales' - pero no sucede lo mismo con la cultura comunicativa: del mismo modo que podemos establecer qué contenidos 'estrictamente lingüísticos' debe conocer un estudiante de cada nivel, debemos ser capaces de determinar qué contenidos culturales comunicativos tienen que saber interpretar y producir en sus interacciones. La razón es puramente lógica: la progresión en ambas esferas determina la competencia comunicativa del hablante, por lo que no hay motivo para no graduar los contenidos culturales. Veamos unos ejemplos extraídos de este apartado del PCIC:

- 1.4.2. Convenciones sociales y comportamientos en la mesa. Fase de profundización. "Convenciones sociales en la mesa relacionadas con la interacción: elogiar un plato y a quien ha cocinado".

- 1.4.3. Establecimientos. Fase de aproximación. "Convenciones sociales y comportamientos: formas de tratamiento con los camareros, formas de pedir".

\footnotetext{
${ }^{12}$ Presentes en el apartado '3.1. Literatura y pensamiento' del PCIC.

${ }^{13}$ Epígrafe 3 de los cuatro que componen el apartado 12 del PCIC.
} 
- 1.5.1. Centros de enseñanza. Fase de profundización. “Convenciones sociales y comportamientos en el contexto educativo en la interacción: formas de dirigirse a un profesor en función del nivel académico y otros factores".

- 1.6. Trabajo y economía. Fase de profundización. "Convenciones sociales y comportamientos relacionados con las entrevistas de trabajo: fórmulas de tratamiento, saludos, movimientos y posturas corporales, concepción y uso del espacio, sistema paralingüístico (signos sonoros, pausas, silencios)".

- 1.7.1. Hábitos y aficiones. Fase de profundización. "Convenciones sociales y comportamientos relacionados con actividades de ocio de carácter social: quedar con amigos".

- 2.1.1. Relaciones sentimentales, familiares y de amistad. Fase de aproximación. "Convenciones sociales en el trato entre amigos y familiares, según la edad, el sexo y la relación establecida: fórmulas para saludar (dar la mano, besar), tratamiento, tono empleado, registro, movimientos y posturas corporales, concepción y uso del espacio, sistema paralingüístico (signos sonoros, pausas, silencios, etc.).

Esta pequeña muestra ejemplifica hechos culturales relacionados directamente con la eficacia comunicativa, los cuales se llevan a cabo a través de la interacción y ponen de manifiesto la imbricación entre la lengua y el entorno social y cultural. Además, la manera en que se realizan varía según la cultura del hablante pero, contra todo pronóstico, el PCIC no explicita cómo se hace esto, lo que repercute negativamente en aquellos docentes cuya cultura de origen es diferente a la española. Ante esta situación, cabe plantearse cuestiones como: ¿no es pertinente concretar qué gestos debe conocer un estudiante de A1 para saludar, puesto que esta función es propia de este nivel?, ¿no hay gestos, fórmulas de tratamiento o rasgos suprasegmentales menos rentables para iniciar una conversación que -al igual que los exponentes lingüísticosno son necesarios hasta niveles superiores?, ¿no es necesario que el estudiante conozca si debe o no compartir información personal -porque en su cultura lo hace- en una entrevista de trabajo o en un trámite administrativo? Obviamente, si pretendemos que el estudiante se comunique de una manera eficaz la respuesta a todas ellas es sí.

Para resumir, concluimos que el componente cultural en el currículo de ELE:

- No tiene una identidad propia: debido a la vaga conceptualización del propio término, los contenidos culturales estén diseminados y mezclados en múltiples apartados, además de que no existe vínculo entre el resto de los subcomponentes y el componente cultural.

- Ha de ser estructurado en base a la distinción 'cultura material' / 'cultura comunicativa': esta organización permite incluir en el currículo aquellos contenidos que influyen directamente en la eficacia comunicativa y mantener, como conocimiento factual, los contenidos externos a la comunicación.

- Puede y debe ser nivelado: hay hechos culturales que repercuten en el progreso de la competencia comunicativa del hablante y por lo tanto han de secuenciarse. 


\section{UNA PROPUESTA A TRAVÉS DE LA PRAGMÁTICA INTERCULTURAL}

En este epígrafe ofrecemos una organización curricular para trasladar el componente cultural, de manera integral y progresiva, a la práctica docente. Intentamos responder, de esta manera, a una de las dificultades que el propio $P C I C$ reconoce en su introducción:

Los profesores, en general, no disponen de la documentación necesaria o de la metodología adecuada que les ayude a determinar qué datos sociales, culturales y sociolingüísticos buscar, y cómo y dónde encontrarlos. De hecho, las descripciones de este tipo de aspectos no son fáciles de elaborar, ya que requieren una respuesta clara a problemas complejos, como acotar el concepto mismo de cultura o desarrollar esquemas manejables con categorías que, más allá de la utilidad que puedan tener para el análisis antropológico, sean relevantes y aplicables en el ámbito de la enseñanza de lenguas (Instituto Cervantes, 2006: 39-40, vol. I).

Como indicamos en el apartado 2.3.1., consideramos que es en la Pragmática donde se manifiesta con mayor claridad la interdependencia entre lengua y cultura, más concretamente en la Pragmática Intercultural. Hernández Sacristán (1999) da un paso más y afirma que, si contrastáramos las categorías pragmáticas de diferentes códigos lingüísticos, estas, por su propia naturaleza, no podrían ser entendidas sin contrastar también los hechos culturales de esas comunidades. Así, concluye:

Comunicación interlingüística es también, por lo que se refiere al menos a las categorías pragmáticas, comunicación intercultural. Sucede, en efecto, que el uso de una categoría pragmática es al mismo tiempo una praxis lingüística y una praxis cultural (Hernández Sacristán, 1999: 26).

Es decir, en las categorías pragmáticas se observa "una naturaleza bifronte lingüística-cultural" (Hernández Sacristán, 1999: 33). Esto se debe a que son parte del sistema lingüístico pero operan sobre el control de las situaciones interactivas de uso, por lo que "gracias a ellas queda modelado un tipo de conducta que, siendo lingüística, es al mismo tiempo valorable en términos sociales" (Hernández Sacristán, 1999: 33). Antes de presentar la propuesta debemos hacer dos consideraciones previas. La primera de ellas es respecto al alcance de la misma. En el diseño curricular de ELE existen dos niveles de concreción: el PCIC es un primer nivel de concreción del $M C E R L$ - puesto que este sentaba las bases de manera general e invitaba a cada lengua a que especificara su currículo-, mientras que el segundo nivel de concreción es el que, tomando como punto de partida el PCIC, se lleva a cabo en cada centro, universidad o academia. Es a este nivel de concreción al que nos dirigimos, ya que en nuestra propuesta tomamos como referencia las unidades del PCIC.

La segunda consideración es respecto a la propia naturaleza de los hechos culturales y a su didáctica. Como vimos, aquellas realidades que pertenecen a la cultura material son saberes enciclopédicos, acumulativos y factuales. Son conocimientos que se tienen -en mayor o menor porcentaje- y cuya didáctica pretende que se amplíen. Por otro lado, la cultura comunicativa tiene que ver con la adecuación a los patrones que rigen la interacción, por lo que no se trata de un conocimiento acumulativo sino interpretativo e interactivo. Además es una realidad que, pese a ser estable, sufre variaciones en función del contexto de uso. Por ello, el progreso no se mide en cantidad sino en efectividad: no es un 'saber' sino más bien un 'saber hacer', una destreza. 
Una vez hechas estas aclaraciones, veamos brevemente cómo podemos diseñar nuestro currículo en aras de incorporar contenidosculturales rentables paralos estudiantes:

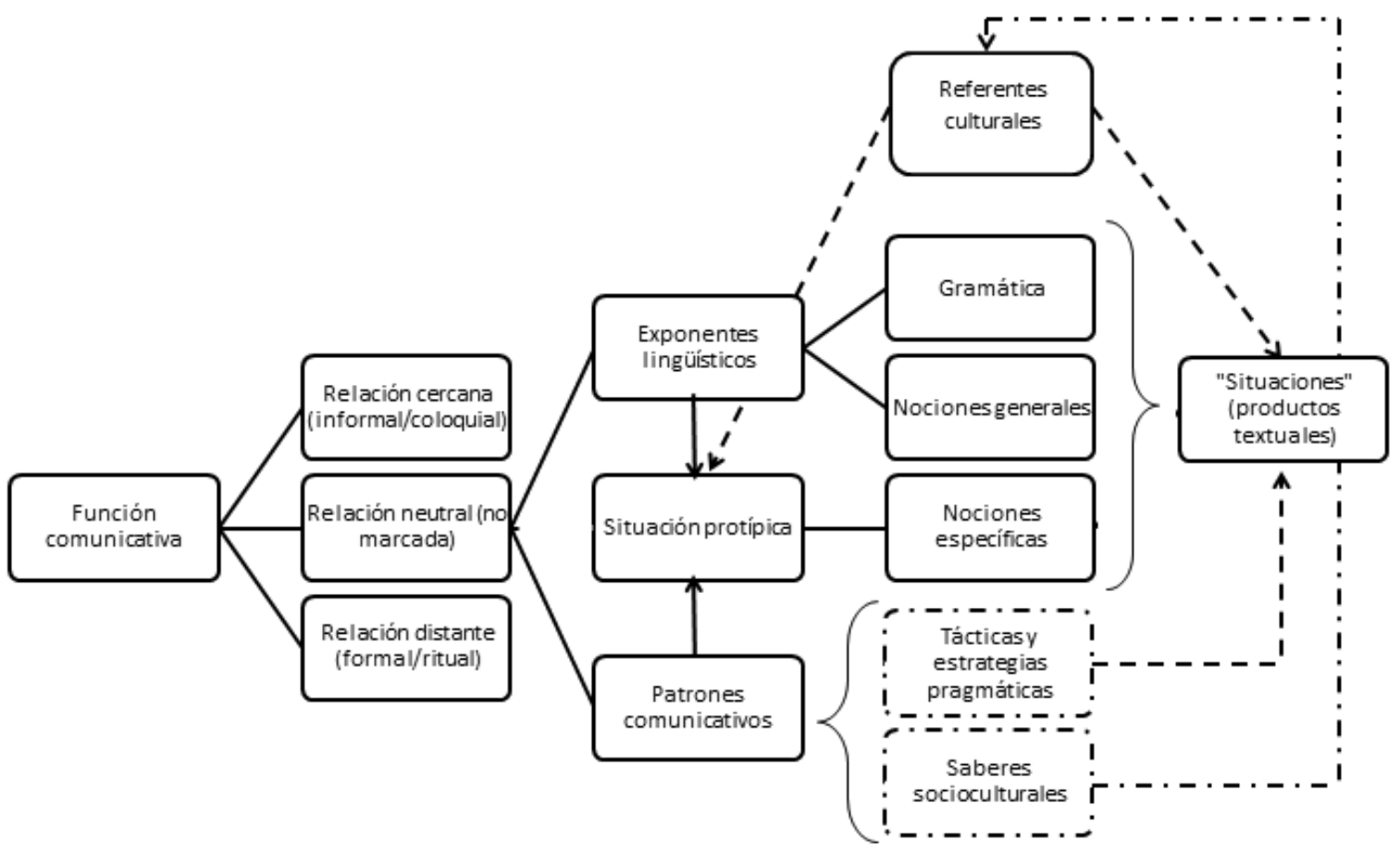

Gráfico 1. Estructura para diseñar un currículo de ELE

\subsection{Vertebración ( $1^{a}$ fase)}

Todocurrículodebe reflejar una concepción del proceso deenseñanza-aprendizaje. En nuestro caso, nos hemos fijado tres objetivos: que esté vertebrado en torno a un único criterio organizador; que sea integrador, es decir, que todos los subcomponentes estén presentes y tengan el mismo tratamiento formal y que sea eminentemente comunicativo, o sea, que el desarrollo del mismo se haga en función de la eficacia comunicativa.

En cuanto a la unidad vertebradora, decidimos tomar las funciones comunicativas porque cumplían con todos los requisitos que habíamos establecido previamente, a saber: que fuera finita; que fuera definible y descriptible, es decir, que fuera un concepto claro respecto al que existiera un cierto consenso en nuestra disciplina; que fuera susceptible de ser nivelado y, por último, que fuera aglutinadora y 'mayéutica', o sea, que propiciara el desarrollo de otras unidades del lenguaje.

\subsection{Nivelación ( $2^{a}$ fase)}

Una de las opciones para nivelar un currículo es dividir el número total de unidades entre los seis niveles y distribuirlas según su grado de complejidad. Para esta propuesta, consideramos que ese método presenta dos inconvenientes: en primer lugar, el criterio de 'más difícil' / 'menos difícil' no es objetivo. En segundo lugar, la función comunicativa no puede ser tratada como una unidad indivisible puesto que dentro de la misma cultura hay diferentes realizaciones según el contexto situacional en el que se desarrolla. A 
este respecto, el grupo CRIT $(2003,2006)$ y Raga Gimeno (2005) consideran el grado de "proximidad" o "lejanía" que exista entre los hablantes como el factor determinante.

En este sentido, consideramos que es necesario que se atienda a cada función desde los tres escenarios posibles -cercanía, neutralidad y lejanía-, por lo que deben ser las funciones en su uso real las que se nivelen y no en sentido abstracto, es decir, descontextualizadas. Como criterio de nivelación hemos de considerar que, si uno de los objetivos es que los estudiantes sean eficaces en su comunicación, debemos recurrir al uso real y por lo tanto distribuir los contenidos según la productividad y la frecuencia de aparición que tengan nuestra cultura comunicativa. Esto significa que un estudiante puede necesitar aprender a hacer un cumplido en un registro coloquial en A2 pero que no sea necesario que acceda a las realizaciones de esta función en un registro formal hasta un B1. De esta manera conseguimos también un aprendizaje circular ya que las funciones se retoman en estadios posteriores.

\subsection{Realización ( $3^{a}$ fase)}

En una interacción cara a cara, una función comunicativa concreta tiene lugar en un contexto situacional específico ${ }^{14}$ y se lleva a cabo a través de dos tipos de emisiones informativas simultáneas: los exponentes lingüísticos, es decir, las unidades lingüísticas que se utilizan convencionalmente con un propósito determinado y los patrones comunicativos, o sea, las unidades culturales que intervienen en la interacción. Estas, como hemos visto, dependen de la cultura comunicativa de la comunidad, lo cual convierte a la cultura comunicativa, en cierta manera, en otro tipo de código.

La perspectiva de la Pragmática Intercultural que considera la función como la realización simultánea de un proceso lingüístico y otro cultural posibilita, a través del uso real, vincular el componente cultural con el resto de los subcomponentes, ya que todos ellos influyen en la comunicación eficaz. Respecto a los exponentes lingüísticos, en aras de proporcionar a los estudiantes realizaciones próximas al uso recomendamos no tomar exclusivamente aquellos que ofrece el PCIC sino recurrir también a los corpora, especialmente el Corpus del español del siglo XXI (Real Academia Española, en línea). Además, las últimas investigaciones sobre disponibilidad léxica sugieren la posibilidad de utilizar guiones en lugar de los centros de interés clásicos, por lo que podría ser una herramienta muy útil en este campo (Cornejo Tomé, 2012).

En cuanto a los patrones comunicativos, seguimos la propuesta de Raga Gimeno (2005) por considerarla especialmente aplicable al campo de la enseñanza de lenguas extranjeras. Este apartado es de suma importancia porque incluye unidades que, como vimos en el apartado 2.3.2., no han sido inventariadas en el PCIC. Se pueden clasificar en seis: máxima de contenido (cantidad y tipo de la información); máxima de veracidad (autoimagen transmitida, uso de las mentiras sociales y de legitimadores

\footnotetext{
${ }^{14}$ En el gráfico, por motivos de espacio, solo hemos desarrollado uno de los tres posibles contextos pero el mismo proceso se repite para los otros dos escenarios -lejanía y cercanía.
} 
externos); máxima de manera ${ }^{15}$ (fórmulas de tratamiento y dirección de los actos de habla); paralenguaje (presencia y énfasis de las características prosódico-informativas), distribución del tiempo (uso de mecanismos de interacción y tipo de secuencias y de turnos) y distribución del espacio (pertinencia del contacto corporal y visual y tratamiento del espacio y de las distancias).

Sin embargo, no se debe caer en el error de suponer que los patrones comunicativos de cada cultura son inalterables puesto que

no existen «comportamientos comunicativos exclusivos». Lo que diferencia a las culturas no es que presenten comportamientos comunicativos completamente diferentes, sino la «extensión» de los mismos (Raga Gimeno, 2005: 169).

En otras palabras, cada situación demanda unos patrones comunicativos y estos pueden alejarse de lo prototípicamente esperable para esa cultura. Como hemos visto, esta variación depende del grado de cercanía o confianza que exista entre los participantes: cuando es mayor que en una situación neutral o 'no marcada' se potencia el grado de proximidad y simetría entre los hablantes, mientras que en una situación de menor confianza o cercanía se realza la distancia y la asimetría. Por este motivo, los patrones comunicativos no pueden enseñarse como una suerte de oposiciones entre comunidades culturales.

La cultura comunicativa española es caracterizada como "próxima y simétrica" (Grupo CRIT, 2003, 2006; Raga Gimeno, 2005) o "de acercamiento" (Briz Gómez, 2007), lo que hace que los patrones se manifiesten de la siguiente manera -en una situación prototípica, esto es, neutral entre los participantes.

\begin{tabular}{|l|l|}
\hline Máxima de contenido & $\begin{array}{l}\text { Intercambio informativo abundante } \\
\text { Información personal y comprometida abundante }\end{array}$ \\
\hline Máxima de veracidad & $\begin{array}{l}\text { Mentiras sociales poco habituales } \\
\text { Transmisión de una imagen propia positiva } \\
\text { Uso de mediadores poco habitual }\end{array}$ \\
\hline Máxima de manera & $\begin{array}{l}\text { Preferencia por los actos de habla directos } \\
\text { Comunicación cooperante }\end{array}$ \\
\hline Paralenguaje & Énfasis elevado: tiene importancia comunicativa \\
\hline $\begin{array}{l}\text { Distribución del } \\
\text { tiempo }\end{array}$ & $\begin{array}{l}\text { Secuencias poco diferenciadas } \\
\text { Turnos cortos } \\
\text { Silencios escasos y breves } \\
\text { Solapamientos habituales } \\
\text { Continuadores habituales } \\
\text { Compleción habitual }\end{array}$ \\
\hline $\begin{array}{l}\text { Distribución del } \\
\text { espacio }\end{array}$ & $\begin{array}{l}\text { Distancias cortas } \\
\text { Contacto corporal admitido } \\
\text { Contacto visual admitido } \\
\text { Expresividad de manos y rostro }\end{array}$ \\
\hline
\end{tabular}

\footnotetext{
${ }^{15}$ Los patrones de la máxima de contenido, veracidad y manera surgen de la reinterpretación de los
} conceptos introducidos por Grice (1975). 
Finalmente, hemos decidido incluir una categoría nueva que hemos denominado 'situaciones prototípicas'. La enseñanza por situaciones tiene una gran tradición (Van Ek, 1975; Slagter, 1979) en el campo de la didáctica de segundas lenguas y consideramos que, en esta concreción curricular, es totalmente pertinente ya que las realizaciones pragmáticas y culturales dependen, en gran medida, del contexto en el que tiene lugar la interacción. Además, su importancia no solo radica en que aglutina la praxis lingüística y la praxis cultural en una sola unidad sino que también sirve para proyectar las nociones específicas.

Hemos definido la situación prototípica como aquella que: refleja la realidad de la cultura meta, es decir, existe en esa comunidad; se lleva a cabo de manera oral; es rentable para el estudiante, o lo que es lo mismo, es muy probable que en sus interacciones con hablantes nativos se encuentre en esa situación y es realizable, o sea, el alumno puede resolverla eficazmente con los recursos lingüísticos y culturales que conoce.

\subsection{Ampliación (4 $4^{a}$ fase)}

Si la propuesta curricular quedara definida exclusivamente en cuanto a los exponentes lingüísticos, los patrones comunicativos y las situaciones prototípicas, se obviarían muchos componentes que influyen en la competencia comunicativa. Asimismo, no se cumpliría uno de los objetivos con los que planteamos nuestra propuesta: "todos los subcomponentes deben estar presentes y tener el mismo tratamiento formal". Por ello, a partir de los tres componentes primarios de la función comunicativa se han de desarrollar otros contenidos específicos.

Pese a que nuestra propuesta sea eminentemente comunicativa e interaccional, valoramos el tratamiento explícito de los contenidos gramaticales en la enseñanza. En la práctica docente, frecuentemente, la discordancia no se produce por los contenidos seleccionados sino por la manera de presentarlos, es decir, el motivo que justifica que los discentes los adquieran. En nuestra propuesta, consideramos que es necesario ampliar los contenidos gramaticales cuando son un factor clave en la realización de una función concreta o en las situaciones en las que la variación en la realización de una función según el grado de cercanía de los hablantes se manifiesta a través de ellos.

Como indicamos en el apartado anterior, una de las utilidades de la situación prototípica es acercar el aprendizaje a interacciones reales de uso. Esta contextualización posibilita la integración de las nociones específicas y las nociones generales de un modo progresivo, rentable y vinculado a las funciones comunicativas, ya que para que la comunicación sea eficaz han de dominar también estas categorías. En este caso, recomendamos que la selección no recurra exclusivamente a inventarios de ELE -como el PCIC - sino que también se consideren los estudios de disponibilidad léxica.

Por último, existen tres apartados en el PCIC-'Tácticas y estrategias pragmáticas', 'Saberes y comportamientos socioculturales' y 'Referentes culturales' cuyo análisis está en la base de esta propuesta curricular. Como vimos en el apartado 2.3.2., la totalidad de 
los referentes culturales y la mayoría de los saberes y comportamientos socioculturales son hechos culturales englobados en la cultura material. Consideramos que, si bien el conocimiento de estas unidades no está vinculado al desarrollo comunicativo, es positivo - para fortalecer la competencia intercultural- que se integren en el currículo a través de las situaciones que hayamos escogido.

En cuanto al componente pragmático, el enfoque de nuestra propuesta lo concibe como parte inseparable de los patrones comunicativos, aunque consideramos que algunos apartados del PCIC -marcadores del discurso, por ejemplo- son más rentables en el siguiente apartado.

\subsection{Destrezas escritas ( $5^{a}$ fase)}

Finalmente, no debemos excluir las destrezas escritas ${ }^{16}$ pese a que esta propuesta curricular parta de las interacciones cara a cara. En todos los productos textuales prevalece una macrofunción por lo que consideramos que la selección se debe hacer teniendo en cuenta el vínculo de esta con la función comunicativa seleccionada, los contenidos gramaticales y pragmáticos y las nociones generales y específicas. Asimismo, este es un espacio idóneo para ampliar contenidos sobre la cultura material.

\section{CONCLUSIONES}

A tenor de lo expuesto a lo largo de este trabajo, concluimos reclamando la necesidad de una identidad propia para el componente cultural. La amplitud del propio concepto no debe desanimarnos en la tarea de definir y distribuir sus contenidos pues, como hemos visto, la integración de estos en la práctica docente repercute positivamente en el proceso de aprendizaje. De esta manera, si el componente cultural adquiere la visibilidad que merece pueden ser minimizados los efectos de procesos psicológicos, sociales e incluso afectivos a los que los estudiantes se enfrentan cuando se sumergen en contextos interculturales.

Sin embargo, la realidad es que el PCIC reserva un espacio marginal para la cultura: tanto la nivelación como la organización de los contenidos culturales son diferentes al resto de los componentes, lo cual dificulta la inclusión de estos en la segunda concreción del currículo. Esta realidad justifica la necesidad de reformular el componente cultural y para ello hemos de partir del propio concepto de cultura.

Ni la caracterización clásica de los hechos culturales en 'highbrow culture' y 'lowbrow culture' ni la ofrecida por el PCIC -'lo estrictamente lingüístico' y 'lo que tiene proyección comunicativa'- esclarecen qué contenidos deben formar parte del currículo y cuáles no. Por el contrario, concebir la cultura como un proceso interpretativo e interactivo y diferenciar entre la cultura material -externa a la comunicación- y la comunicativa -la cual se manifiesta a través de los patrones comunicativos- permite

\footnotetext{
${ }^{16}$ En este apartado consideramos exclusivamente los productos textuales de transmisión escrita ya que los géneros de producción oral se encuadran dentro de la situación prototípica.
} 
seleccionar los contenidos en base a un criterio sólido y estable. Además, esta concepción vincula el componente cultural con el resto de los subcomponentes por lo que hace posible nivelar los contenidos escogidos.

Por último, la estructura actual del PCIC obstaculiza la labor de los centros y de los docentes que quieren integrar una cultura rentable en sus clases de ELE. Es obvio que el PCIC necesita una restructuración -no exclusivamente respecto al componente cultural- y que este no será un proceso sencillo ni inmediato. Por este motivo, ofrecemos una estructura aplicable a la segunda concreción del currículo, que, si bien es un primer paso, debe facilitar la integración del componente cultural a través de las funciones comunicativas.

\section{BIBLIOGRAFÍA}

Bachman, L. F. (1990 [1995]): “Communicative Lenguage Ability", en M. Llobera (coord.) (1995) Competencia comunicativa. Documentos básicos en la enseñanza de lenguas extranjeras, Madrid, Edelsa: 105-127.

Briz Gómez, A. (2007): "Para un análisis semántico, pragmático y sociopragmático de la cortesía atenuadora en España y América", LEA, 29, 1, 5-40.

Bueno, A. (1995): “Sociolinguistic and Sociocultural Competence”, en N. McLaren y D. Madrid (eds.) (1995) A Handbook for TEFL, Alcoy, Marfil: 345-374.

Byram, M. y Fleming, M. (1998 [2001]): Perspectivas interculturales en el aprendizaje de idiomas, Cambridge, Cambridge University Press.

Canale, M. (1983 [1995]): "From communicative competence to communicative language pedagogy", en M. Llobera (coord.) (1995) Competencia comunicativa. Documentos básicos en la enseñanza de lenguas extranjeras, Madrid, Edelsa: 63-81

Canale, M. y Swain, M. (1980): “Theoretical bases of communicative approaches to second language teaching and testing", Applied Linguistics, 1, 1-47.

Carcedo González, A. (1996): “Cultura y patrones de comportamiento: su integración en la enseñanza de la lengua", en A. Celis et alii (coords.) (1996) Lengua y cultura en la enseñanza de español a extranjeros: Actas del VII Congreso de ASELE. Cuenca, Ediciones de la Universidad de Castilla-La Mancha: 165-173.

Celce-Murcia, M.; Dörnyei, Z. y Thurrell, S. (1995): “A pedagogically motivated model with content specifications", Issues in Applied Linguistics, 6, 5-35.

Cenoz, J. (2000): “La adquisición de la competencia pragmática: implicaciones para la enseñanza de lenguas extranjeras", en S. Salaberri (ed.) (2000) Lingüística Aplicada a la Enseñanza de Lenguas Extranjeras, Almería, Universidad de Almería: 379-405

Consejo de Europa (2002): Marco común europeo de referencia para las lenguas: aprendizaje, enseñanza, evaluación, Madrid, MECD-Grupo Anaya. 
Cornejo Tomé, C. (2012): “La utilización de guiones (scripts) como estímulos temáticos en los estudios de disponibilidad léxica", Interlingüística, 22, 459-474.

Duranti, A. (2000): Antropología lingüística, Madrid, Cambridge University Press.

Escandell Vidal, Ma . V. (2006): Introducción a la pragmática (2a ed.), Barcelona, Ariel.

Estévez Coto, M. y Fernández de Valderrama, Y. (2006): El componente cultural en la clase de E/LE, Madrid, Edelsa.

Geertz, C. (1973 [1992]): La interpretación de las culturas, Barcelona, Gedisa.

González Plasencia, Y. (en prensa): “Los actos de habla indirectos desde la óptica de la cultura comunicativa", ForoELE.

Goodenough, W. H. (1971): “Cultura, lenguaje y sociedad”, en J. S. Kahn (ed.) (1975) El concepto de cultura: textos fundamentales, Barcelona, Anagrama.

Grice, P. (1975): “Logic and conversation”, en P. Cole y J. L. Morgan (eds.) (1975) Syntax and Semantics (Vol. 3). Speech Acts, New York, Academic Press: 41-58.

Grupo CRIT (2003): Claves para la comunicación intercultural, Castellón, Publicaciones Universitat Jaume I.

Grupo CRIT (2006): Culturas cara a cara, Madrid, Edinumen.

Gudykunst, W. B. (1987): “Cross-Cultural comparisions”, en C. R. Berger et alii (eds.) (1987) Handbook of Communication Science. Londres, Sage: 847-889.

Gudykunst, W. B. (ed.) (2005): Theorizing about intercultural communication, Thousands Oak, CA, Sage.

Guillén Díaz, C. (2004): “Los contenidos culturales”, en J. Sánchez Lobato e I. Santos Gargallo, (eds.) (2004) Vademécum para la formación de profesores, Madrid, SGEL: 835-851.

Gumperz, J. J. y Bennett, A. (1981): Lenguaje y cultura, Barcelona, Anagrama.

Harris, M. (1968): The rise of anthropological theory. A history of theories of culture, New York, Crowell.

Hernández Sacristán, C. (1999): Culturas y acción comunicativa. Introducción a la pragmática intercultural, Barcelona, Octaedro.

Hofstede, G. (1980): Culture's consequences: International Differences in work-related values, Beverly Hills Ca, Sage Publications.

Hofstede, G. (1999): Cultura y organizaciones: el software mental, Madrid, Alianza Editorial.

Humboldt, W. von (1990): Sobre la diversidad de la estructura del lenguaje humano y su influencia sobre el desarrollo espiritual de la humanidad, Barcelona, Anthropos.

Iglesias Casal, I. (2003): “Construyendo la competencia intercultural: sobre creencias, conocimientos y destrezas", Carabela, 54, 5-29.

Instituto Cervantes (2006): Plan Curricular del Instituto Cervantes. Niveles de referencia para el español, Madrid, Biblioteca Nueva. 
Instituto Cervantes (2014): El español: una lengua viva, http:/ / cvc.cervantes.es/lengua/ anuario/anuario_02/lujan/p02.htm (consultado en mayo de 2015).

Kahn, J. S. (1975): El concepto de cultura: textos fundamentales, Barcelona, Anagrama.

Kim, Y. Y. (1988): Communication and cross-cultural adaptation: An integrative theory, Philadelphia, Multilingual Matters.

Kim, Y. Y. (1995): “Cross-cultural adaptation: an integrative theory”, en R. L. Wiseman (ed.) (1995) Intercultural communication theory. Thousands Oak, CA, Sage: 170193

Kramsch, C. (1998 [2001]): “El privilegio del hablante intercultural”, en M. Byram y M. Fleming (eds.) (2001) Perspectivas interculturales en el aprendizaje de idiomas, Cambridge, Cambridge University Press: 23-38.

Mead, G. H. (1962 [1999]): Espíritu, persona y sociedad, Barcelona, Paidós.

Miquel, L. y Sans. N. (1992 [2004]): “El componente cultural: un ingrediente más en las clases de lengua", Red ELE, 0. Disponible en: http://www.mecd.gob.es/ redele/revistaRedEle/2004/primera.html

Oberg, K. (1960): “Cultural shock: Adjustment to New Cultural Enviroments”, Practical Antropology, 7, 177-182.

Oliveras, A. (2000): Hacia la competencia intercultural en el aprendizaje de una lengua extranjera, Madrid, Edinumen.

Raga Gimeno, F. (2005): Comunicación y cultural. Propuestas para el análisis transcultural de las interacciones comunicativas cara a cara, Madrid, Iberoamericana Vervuert.

Real Academia Española: Banco de datos: (CORPES XXI) [en línea]. Corpus del español del siglo XXI (CORPES).

Rodrigo Alsina, M. (1999): Comunicación intercultural, Barcelona, Anthropos.

Sapir, E. (1949): Selected writings in language, culture and personality, Berkeley, University of California Press.

Slagter, J. P. (1979): Un Nivel Umbral, Estrasburgo, Publicaciones del Consejo de Europa. Trujillo Sáez, F. (2005): “En torno a la interculturalidad: reflexiones sobre cultura y comunicación para la didáctica de la lengua", Porta Linguarum, 4, 23-39.

Van Ek, J. A. (1975): The Threshold Level in a European Unit/Credit System of Modern Language Learning by Adults, Estrasburgo, Publicaciones del Consejo de Europa.

Vázquez, G. (2012): “El desarrollo del componente estratégico dentro del marco actual de la didáctica de ELE: estado de la cuestión y perspectivas", en Revista Internacional de Lenguas Extranjeras, 1, 181-212.

Whorf, B. I. (1956): Language, thought and reality, Cambridge-Massachusetts, The M.I.T. Press. 